\title{
All-On-Four Concept in Implant Dentistry: A Literature Review
}

\section{Durkan $\mathrm{R}^{1}$ and Oyar $\mathrm{P}^{*_{2}}$}

\author{
${ }^{1}$ Department of Prosthodontics, Faculty of Dentistry, Afyon Kocatepe University, Afyonkarahisar, Turkey \\ ${ }^{2}$ Health Services Vocational High School, Department of Dental Prostheses Technology, Hacettepe University, An- \\ kara, Turkey
}

${ }^{*}$ Corresponding author: Oyar P, DDS, Ph.D., Associate Professor, Health Services Vocational High School, Department of Dental Prostheses Technology, Hacettepe University, D-Blok, 3. Kat, 06100 Sihhiye, Ankara, Turkey, E-mail: poyar73@gmail.com

Citation: Durkan R, Oyar P (2017) All-On-Four Concept in Implant Dentistry: A Literature Review. J Dent Oral Care Med 3(2): 205. doi: 10.15744/2454-3276.3.205. doi: 10.15744/2454-3276.3.205

Received Date: August 9, 2017 Accepted Date: September 20, 2017 Published Date: September 22, 2017

\begin{abstract}
The implant technology developed as the all-on-four concept is offered as an alternative to conventional implant applications. In the all-on-four technique, 4 implants are placed in the interforaminal region in the mandible and in the pre-maxillary region in total edentulism cases. This application is based on the placement of two of these implants to the anterior region so as to be perpendicular to the occlusal plane, and on the placement of two implants to the posterior region so as to be inclined $30-45^{\circ}$. These implants are ensured to function by making immediate fixed complete denture over them. The patient is administered with permanent fixed full arch restoration 3 months later. The reduction of surgical procedures and the functioning of immediate dentures are important by means of this system. The purpose of this study is to compile the clinical and research articles regarding the all-on-four implant concept, to determine the basic principles of this concept, and to present the advantages and disadvantages of the method. The all-on-four concept can be an alternative and potential treatment method especially in atrophic edentulous jaws, its routine clinical practices can be performed, but it is necessary to increase long-term clinical controlled studies.
\end{abstract}

Keywords: All on four concept; Dental implant; Tilted implant

\section{Introduction}

In patients with maxillary and/or mandibular total edentulism, dental implant treatment is a treatment model which has been accepted scientifically, and long-term studies have been carried out on this subject [1-6].

In recent years, putting implants into immediate function by making implant-supported fixed full-arch restorations and the fact that patients do not remain edentulous have become gold standard in dental implantology [7-10].

It is observed in the literature that successful prostheses are made using 6-8 implants in the maxilla and 6 implants in the mandible and applying posterior cantilever extension where it is necessary, in these treatments [3]. In full arch fixed implant-supported prostheses, patients achieve the comfort, aesthetics, phonetics and functional chewing effectiveness in their natural teeth [11-13]. The clinical and hygiene controls can be easily performed especially in full arch screw-retained fixed prostheses [3].

However, in these treatments, the presence of overly baggy maxillary sinuses for maxilla restricts the implant placement in the posterior region especially in patients with excessive resorbed crests [14-16]. Pterygoid, tuber, and zygomatic implants can be placed in the posterior region, and autogenous or heterogeneous bone grafts can be used for the solution of this [8]. However, extended surgical operations, deterioration of patient comfort and the risks of surgical complications increase in that time. 8 The posterior implant treatment also constitutes a disadvantage in patients with resorbed mandible with a mandibular nerve located at the top of the alveolar crest. Nervous repositioning, graft applications, short and/or angled implant applications also bring along surgical and patient based disadvantages [17-19].

The all-on-four implant technology has been developed to prevent all these disadvantages [11]. The technique [20] that has gained popularity in full arch edentulism cases in recent years was presented as a modern technique in implant-denture rehabilitation by Malo for the first time in 2003 and the all-on-four concept that began to be used in atrophic full arch mandibular and in the maxilla in 2005 has emerged [7,8,21] Accordingly, the purpose of full arch implant application is to reduce the cantilever extensions by removing anatomic region limitations [22]. Implants are positioned in the pre-maxillary region in the maxilla as median and in 
the inter-foraminal region in the mandible $[23,24]$.

Implants are placed in different regions related to anterior and posterior implant sites. Anterior implants are placed to the lateral incisor sites or canine/first premolar region, posterior implants are placed to the second premolar or first molar region [7,25,26].

The fact that full arch screw-retained fixed prostheses and immediate dentures made on a total of 4 implants including two implants that are orthogonally placed to the occlusal plane in the anterior region and two implants that are placed in the posterior region with a mesial angle of 30-45 in edentulous maxillary and / or mandibular jaws are constructed and immediately loaded (after a surgery of 8-48h), and the construction of permanent fixed prosthesis after a 3-month period constitute the basis of the all-on-four concept [25,27-29].

The fact that the distribution of the forces is biomechanically sufficient with 4 implants placed at right angles and positions [30], the use of longer implants for the posterior region $(\geq 13 \mathrm{~mm})$ and accordingly increase in bone anchorage and placement in the correct biomechanical position, and ensuring high primary stability are regarded as the advantages of the all-on-four concept [28,29,31-33]. The use of computer-assisted methods (computer assisted method-CAM and computer assisted surgery-CAS) in the planning and implant surgery, surgical planning and guide plates are made digitally, increase in the implant success rate, fewer complications and easy post-surgical period for the patients are among the most important features of this concept [34]. In addition, edentulous jaw type, gender, and implant placement sites do not affect the treatment plan and do not biomechanically cause stress increase in the implant-bone connection [35-38].

Nobel Speedy or the Branemark Novum ${ }^{\circledR}$ System (Nobel Biocare AB, Goteborg, Sweden), Branemark System Mk III Ti Unite $[39,40]$ dental implants are generally used in the literature for this concept $[3,7]$. However, different implant systems (Winsix; Bio SA Fin, Ancona, Italy and 2008-Nobel Active) have been used successfully in recent years [26,40,41].

In implant supported dentures, general protocol is formed by placing implants parallel to the anterior maxilla and mandible. In implant-supported fixed dentures, masticatory forces are distributed evenly over the implants, and the loads to be on posterior cantilever extensions are shared on many anterior implants. For this purpose, it is aimed to distribute the loads by increasing the number of maxillary and mandibular anterior implants. However, it was stated in some studies that more loads are formed on the prosthesis in the extensions applied to the distal portion without considering the number of implants placed in the anterior region, the forces are absorbed by the distal implants, and the number of implants does not affect it $[1,42]$.

Many researchers wrote articles about the all-on-four implant concept. Therefore, the purpose of this review is to summarize articles written about all-on-four, to make comparisons between them, to form the advantages and disadvantages of the method from the literature review, and to evaluate the all-on-four concept in terms of osseointegration and crestal bone resorption.

\section{Material and Methods}

In this review, studies involving maxilla and mandible applications of the current all-on-four technique, its advantages and disadvantages, crestal bone loss, and osseointegration and clinical survival rates were examined. In the review performed in Pubmed and Cochrane Library between 2000 and 2015, Full arch implant-supported prosthesis, 4-implant full arc, osseointegration, inclined implant, total edentulism and different combinations in which some of these keywords were used together were chosen. 100 of the articles achieved from the review were evaluated with respect to the subject we examined.

The inclusion criteria for articles were as follows: (1) Articles were related to the all-on-four concept, and (2) abstracts were obtained when the full texts could not be obtained. Articles about conventionally full arch implant usage for edentulous arches were excluded from the review.

\section{Results and Discussion}

100 articles were compiled in the PubMed review. 43 of these articles were found to be suitable for the inclusion criteria. Of these, 30 were laboratory studies, 6 were clinical studies, 4 were case reports, and 3 were review articles. The majority of studies were in vivo clinical follow-up studies. Two studies were carried out with respect to the electromyographic activity of masticatory muscles of the all-on-four concept [29,43], and stress analyses in 4 articles, Three clinical studies involved clinical survival rate.

The all-on-four treatment concept is an attempt to address some objectives such as experienced surgical and restorative team, careful patient selection, short treatment intervals and reduction of surgical procedures by providing relatively straightforward, predictable treatment option for edentulous patient.

Electromyographic evaluation of masticatory muscles: There are 1 clinical study and 1 literature review in this regard.10 of 26 patients aged between 50-74 were administered with mandibular all-on-four - maxillary complete denture [29] 8 maxillarymandibular all-on-four implant-supported fixed prostheses were made. 8 patient with complete dentures were selected as the control group. Masseter and temporalis muscle, maximum voluntary teeth clenching and during unilateral gum chewing were examined with EMG. The neuromuscular balance was found to be good in all patients during clenching. Similar results were obtained in all groups during mastication, in mastication frequency and working side muscle activities. In EMG parameters, 
there was no significant difference between all-on-four implant-supported prostheses and the reference subjects. The all-on-four technique was proposed to be used functionally in edentulous patients indicating that mastication activity is low in complete dentures [29]. While Van lierde et al. were satisfied with $87 \%$ of the prostheses in total in their case-controlled study in which they made all-on-four implant-supported fixed dentures to the mandible and maxilla in 15 patients, and 7 months later they examined them in terms of phonetic characteristics and oromyofunctional behaviour, distortions occurred in the utterance of /s/, /z/, /r/,/d/ and $/ \mathrm{t} /$ letters. Oromyofunctional behavior was found to be normal. It is recommended to begin the speech therapy to strengthen the movements of the tongue immediately after the treatment [25].

Stress analysis: In Cidade et al.'s study [21] in which $100 \mathrm{~mm}$ bilateral cantilever implants with an angle of $15^{\circ}$ and $8 \mathrm{~mm}$ bilateral cantilever implants with an angle of $35^{\circ}$ in maxillary posterior inclined implants were compared in terms of stress by the photoelastic stress analysis, it was observed that the transmission of distal loads coming to cantilever to the inclined implant $35^{\circ}$ inclined implants, reducing the stress and increasing inclination were the advantages. However, it was stated that the stress increased in low inclined implants but it was not harmful, and placement was easy. Apical stresses in implants with $15^{\circ}$ of inclination and cervical stress at $35^{\circ}$ occur when cantilever effect is not taken into account. In the two-way finite element analysis performed to determine the stress caused by the splinting of the implants around the marginal bone of the implant especially in inclined posterior implants, two $13 \mathrm{~mm}$ implants splinted by a titanium beam around the bone, $16 \times 3 \mathrm{~mm}$, distal cantilever $7 \mathrm{~mm}$ in length and which the distal implant (13 or $19 \mathrm{~mm}$ ) was tilted 45 degrees and supported the distal end of the cantilever. A force of $50 \mathrm{~N}$ was applied via the beam. It was emphasized that distal inclined implants did not cause stress increase when they were splinted, marginal stresses were reduced along with the use of the longer posterior implant [31]. In Bevilacqua's 3-dimensional (3-D) finite element analysis which was performed to determine the stresses on the periimplant bone in vertical and inclined implants in the maxilla, 4 different configurations were evaluated with the distal implants inclined $0,15,30$, and 45 degrees; cantilever extensions were 13, 9,5 , and $0 \mathrm{~mm}$, respectively. A vertical load $(150 \mathrm{~N})$ was applied to the distal portion of the posterior cantilevers. The maximum stresses recorded in periimplant bone for the vertical implants were 75.0 MPa (distal implants), 35.0 MPa (mesial implants), and 95.0 MPa for the metal frameworks. It was found that stresses were reduced in the periimplant bone and prosthesis in the fullarch fixed prostheses made by the splinting of the implants in the atrophic maxilla, and tilted distal implants, with consequent reduction of the posterior cantilevers [44]. Baggi tried to determine the possible risks of excessive stress and unbalanced load transfer mechanisms and to identify the main biomechanical factors affecting loading transmission 2 vertical mesial implants and 2 tilted distal implants (at a 30-degree angle), and the second used vertical implants that fulfilled platform switching concepts. The cantilever length, the implant design and positioning, and the bone's mechanical properties and morphology can affect both load transmission mechanisms and bone overloading risks in complete-arch restorations supported by 4 implants [3]. In the study by Takahashi, use of 4 implants or inclined implants increased stress on the peri-implant cortical bone. However, when used in conjunction with a short cantilever, inclined implants decreased stress on the peri-implant cortical bone. Edentulous mandible implants were splinted with a superstructure [45] Fixed support prostheses on four implants with a cantilever length of $10 \mathrm{~mm}$ properly resist occlusal loading [46].

Clinical studies: 6 clinical studies carried out according to the all-on-four technique were examined. The cumulative survival rates are reported as $92.2 \%$ and $100 \%$ for the all-on-four concept $[7,8,14,20,23,47]$. Malo et al. [7] reported that marginal bone resorption was low. Malo et al. [8] 128 implants were placed in 32 patients, and at the end of a 1-year follow-up in maxillary prostheses, the1-year cumulative survival rate was $97.6 \%$, marginal bone loss was $0.9 \mathrm{~mm}$ in the implant/abutment junction region. In a retrospective study, the mean annual marginal bone loss was determined at the level of $1.2 \pm 0.7 \mathrm{~mm}$ in 189 patients in which implants (NobelSpeedy concept implant, Nobel Biocare) were placed and it was identified that the marginal bone level was preserved. Furthermore, the total success rate was observed to be $98.9 \%$ [23]. Malo along with computer-guided flapless implant surgery using computer tomography and CAD-CAM technology in surgical planning and making a custom surgical template, at the end of 6 and 21 months clinical follow-up by making 18 maxilla and 5 mandibles full arch dentures, the average marginal bone loss was $1.9 \mathrm{~mm}$ and implant tilting is a viable treatment modality [30]. In their study, Balshi et al. achieved high success rates in patients they treated with Branemark System implants and reported that this method could be an alternative to other treatment protocols. It was determined that there was no difference in axial and tilted implants in terms of osseointegration [7]. Immediate loading was made by making 4 implant-screw retained single jaw all-on-four concept fixed prostheses. Anterior implants were placed to the 2 nd incisor tooth regions perpendicular to the alveolar crest, posterior implants were placed at the 2 nd premolar alignment at a distance from the alveolar nerve in the mandible and the sinus in the maxilla with an angle of 300 [25]. No complication was encountered in screw retained full arch fixed dentures over the all-on-four implant (Winsix; BioSAFin, Ancona, Italy) made using a digital body scan measurement system in the construction of permanent prosthesis, and the marginal bone loss was less than $1.5 \mathrm{~mm}$. insertion torque of at least $40 \mathrm{~N} . \mathrm{cm}$. [26] 3.8- $4.5 \mathrm{~mm}$ in diameter and $13 \mathrm{~mm}$ in length and $4.5 \mathrm{~mm}$ in diameter and 13-15 mm in length Nobel Active implant (Nobel Biocare, Gothenburg, Sweden) was used [48] (Table 1). Marginal bone loss was found to be $0.14 \mathrm{~mm}$ in all-on-four implant prostheses made using platform switch taper implants, only 2 implants failed [35]. Despite the need for at least $4.0 \mathrm{~mm}$ implant diameter to ensure maximum anchorage especially in the mandibular posterior region for the All-on-Four concept it was emphasized in the evaluation of Nobel Active implant with Narrow neck $3.5 \mathrm{~mm}$ diameter that this method could be a new standard [8,24,49]. In the meta-analysis carried out by Menini et al. [14] by including 11 studies in which immediate loading was performed by making maxillary full-arch implant-supported dentures containing upright and tilted implants and at least 1 year clinical follow-up of which were performed, a total of 1,623 implants (778 
tilted, 845 upright) were conducted to 324 patients into the maxilla. 17 tilted and 16 upright implants were lost in the first year. There was no significant difference in terms of failure between the implants. The cumulative survival rate was $97.97 \%$. There was no difference between tilted and upright implants in terms of marginal bone loss at the end of 1-year clinical follow-up in 6 studies, and peri-implant bone resorption was found to be $0.75 \mathrm{~mm}$ (tilted, $0.77 \mathrm{~mm}$; upright, $0.73 \mathrm{~mm}$ ) It was reported that angular implants were successful for short term in maxillary applications, but there was a need for long-term studies to be carried out with respect to tilted and upright implants (Table 1). The most intensively observed complication was the acrylic temporary prosthesis fractures. The prosthesis was suggested to be strengthened with a metal to prevent them. It was also emphasized that bruxism and eating habits cause complication [12]. In the meta-analysis involving 10 studies ( 3 retrospective and 7 prospective), a total of 462 patients were administered with 470 immediately loaded prostheses (257 in the maxilla, 213 in the mandible), supported by a total of 1,992 implants (1,026 upright and 966 tilted). 25 implants failed in the first year. There was no prosthesis failure, implant circumference crestal bone loss did not create a difference in the axial and tilted implants, and the patients were satisfied with their prostheses in terms of aesthetics, function, and phonetics [20]. In the meta-analysis in which 13 studies were examined, the average survival rates of 4,804 implants at the end of 36 months for implants and prostheses were 99.0 1 1.0\% and 99.9 $10.3 \%$, respectively. Maxilla-mandibular and axial-tilted implant difference was not significant [39]. In the clinical controlled study of Francetti et al.,[50] fixed prostheses (CAD-CAM Procera ${ }^{\circledR}$ system (Nobel Biocare AB) were made at the end of 4-6 months, final torque of 40 to $50 \mathrm{Ncm}$ using abutments (Multi-Unit Abutments-MUA, Nobel Biocare AB) and making mandibular implantsupported fixed full arch prostheses (All-on-Four ${ }^{\circledast}$, Nobel Biocare AB, Göteborg, Sweden). It was reported that the patients were satisfied aesthetically and functionally, the marginal bone loss did not create a significant difference between axial and tilted implants during at least one year of observation. Acrylic prosthesis fractures occurred in the use of 4-6 months especially in male patients by 11\%, Malo et al. observed acrylic fractures as 27\% [7] (Table 1). Francetti et al. [51] found that this treatment method could be used safely between axial and tilted implants and between maxilla and mandible in terms of the peri-implant bone level at the end of a clinical follow-up in the prostheses they made using $4 \mathrm{~mm}$ in diameter Nobel Biocare implants. It was emphasized that peri-implant mucositis complications in $30.2 \%$ of patients and peri-implantitis prosthetic complications in $10.4 \%$ of patients did not reversibly affect the implant success rates, biological and technical complications were less in the medium term, but there was a need for long-term studies [52].

\begin{tabular}{|c|c|c|c|}
\hline Investigator and study & $\begin{array}{l}\text { Number of patients - } \\
\text { Number of implants }\end{array}$ & Follow-up period & $\begin{array}{c}\text { Survival rate of } \\
\text { implants- prostheses, \% }\end{array}$ \\
\hline $\begin{array}{l}\text { Malo } 2003 \\
\text { retrospective }\end{array}$ & $44-176$ & 6 months & $98.2-100$ \\
\hline $\begin{array}{l}\text { Malo } 2006 \\
\text { retrospective }\end{array}$ & $46-189$ & 1 years & 98.9 cumulative \\
\hline Malo 2007 preliminary & $23-92$ & 13 months & 97.8-100 cumulative \\
\hline Malo 2012 & $142-227$ & 2 years & \\
\hline Graves & $276-$ & 16 months & \\
\hline $\begin{array}{l}\text { Balshi } 2014 \\
\text { retrospective }\end{array}$ & $152-800$ & 6 years & $97.3-99.0$ \\
\hline Babush 2013 & -227 & 3 years & 98.7 cumulative \\
\hline $\begin{array}{l}\text { Babush } 2011 \\
\text { retrospective }\end{array}$ & $165-709$ & 29 months & 99.6 cumulative \\
\hline Babush 2016 & 169 & 36 months & 99.8 \\
\hline Gherlone & $14-56$ & 1 years & 100 cumulative \\
\hline Van Lierde & $20-80$ & 4 years & 100 \\
\hline $\begin{array}{c}\text { Francetti } 2008 \\
\text { prospective }\end{array}$ & $62-$ & 22.4 months & 100 cumulative \\
\hline $\begin{array}{c}\text { Francetti } 2012 \\
\text { prospective }\end{array}$ & $47-196$ & 5 years & 100 cumulative \\
\hline $\begin{array}{c}\text { Francetti } 2015 \\
\text { retrospective }\end{array}$ & 86- 380 & 65.36 months & \\
\hline Mozzati & -227 & 3years & $98.7-100$ \\
\hline
\end{tabular}

Table1: Clinical studies examining the all-on-four implant and the evaluation of prosthesis success rates

Case reports: Maló et al. [7] presented the first clinical case report of an all-on-four dental implant in the literature. The atrophic full arc was firstly used in the mandible and in the maxilla in 2005. Ferreira et al. [1] stated that the bone structure which is intense in the interforaminal region in the mandible increased the implant success rates despite the high trabecular structure of the maxillary bone. $94 \%$ success in the mandible and $92.8 \%$ success were achieved at the end of the 1-year follow-up in maxillary and mandibular all-on-four implants applied in a patient. Peri-implantit, peri-implant mucositis or mobility were not observed at the end of a 2-year follow-up in all-on-four implant treatment performed in the mandible along with free fibula flap in a postoncologic patient using 3D computer-assisted treatment planning and guided surgery [53]. No complication was found in 
the prosthetic and implants at the end of a 2-year follow-up in all-on-four concept treatment performed in the mandible with two zygomatic (Zygoma Implants RP Machined/Nobel Speed-Nobel Biocare) and two anterior implants and regular platform implants in accordance with the all-on-four concept to the extreme atrophic maxilla of the 55-year-old patient [1]. All-on-four implants (TekkaInkone) were placed to the excessively resorbed mandible, where the mental foremen were at the crestal bone level, of a 58 -year-old patient, and posterior implants were placed to the mental foremen with a mesial angle of $30^{\circ}$. It was observed that the treatment was successful at the end of the 1-year follow-up of the patient administered with mandibular fixed prosthesis and maxillary conventional complete denture [33]. Four $4.3 \times 13 \mathrm{~mm}^{2}$ posterior implants were placed in a 59 -year-old patient by 30 distally inclined with implants (Replace Select Yorba Linda, CA) torqued to $45 \mathrm{Ncm}$ (4-mm height) (Nobel Biocare), and there was no prosthetic and implant complication in a 1 year follow-up in the mandibular prosthesis made with abutments and posterior 17 angled abutments [40]. It was reported that success was achieved in a 1-year follow-up in the patient treated with maxillary all-onfour concept surgery and prosthetic combination by computer-assisted planning as flapless in a patient [27].

According to the results obtained from the review made based on peer-reviewed data, the implementation of immediate loading procedures, especially in the atrophic maxilla and/or mandibular full-arch fixed prostheses along with the all-on-four concept, provides a great advantage for the patient and physician. The use of smaller numbers of implants compared to conventional implant applications and the fact that surgical operations have been shortened increase the use of the technique. Although 5-year follow-ups are available in clinical controlled studies, routine clinical use and clinical performance evaluation of the all-on-four concept can be performed more clearly along with the long-term follow-up studies of 10 years or more.

\section{Conclusion}

The all-on-four concept can be an alternative and potential treatment method especially in atrophic edentulous jaws, its routine clinical practices can be performed, but it is necessary to increase long-term clinical controlled studies.

\section{References}

1. Ferreira EJ, Kuabara MR, Gulinelli JL (2010) "All-on-four" concept and immediate loading for simultaneous rehabilitation of the atrophic maxilla and mandible with conventional and zygomatic implants. Brit J Oral Maxillo Fac Surg 48: 218-20.

2. Misch CE (2008) Comtemporary implant dentistry ( $3^{\text {rd }}$ Edn) Mosby, Elsevier, USA.

3. Balshi TJ, Wolfinger GJ, Slauch RW, Balshi SF (2014) A retrospective analysis of 800 Brånemark System implants following the All-on-Four ${ }^{\mathrm{Tw}}$ protocol. J Prosthodont 23: 83-8.

4. Papaspyridakos P, Chen CJ, Chuang SK, Weber HP, Gallucci GO (2012) A systematic review of biologic and technical complications with fixed implant rehabilitations for edentulous patients. Int J Oral Maxillofac Implant 27: 102-10.

5. Malhotra AO, Padmanabhan TV, Mohamed K, Natarajan S, Elavia U (2012) Load transfer in tilted implants with varying cantilever lengths in an All-on-Four situation. Aust Dent J 57: 440-45.

6. Crespi R, Vinci R, Capparé P, Romanos GE, Gherlone E (2012) A clinical study of edentulous patients rehabilitated according to the "all on four" immediate function protocol. Int J Oral Maxillofac Implants 27: 428-34.

7. Malo P, Rangert B, Nobre MA (2013) "All-on-Four" immediate-function concept with Branemark System implants for completely edentulous mandibles: A retrospective clinical study. Clin Implant Dent Relat Res 5: 2-9.

8. Malo P, Rangert B, Nobre MA (2005) All-on-4 immediate-function concept with Branemark System implants for completely edentulous maxillae: A 1-year retrospective clinical study. Clin Implant Dent Relat Res 7: S88-94.

9. Agliardi E, Clerico M, Ciancio P, Massironi D (2010) Immediate loading of full-arch fixed prostheses supported by axial and tilted implants for the treatment of edentulous atrophic mandibles. Quintessence Int 41: 285-93.

10. Butura CC, Galindo DF, Jensen OT (2011) Mandibular all-on-four therapy using angled implants: a three year clinical study of 857 implants in 219 jaws. Dent Clin North Am 55: 795-811.

11. Asawa N, Bulbule N, Kakade D, Shah R (2015) Angulated Implants: An Alternative to Bone Augmentation and Sinus Lift Procedure: Systematic Review. J Clin Diagnos Res 9: Z10-3.

12. Tealdo T, Bevilacqua M, Pera F, Menini M, Ravera G, et al. (2008) Immediate function with fixed implant-supported maxillary dentures: a 12-month pilot study. J Prosthet Dent 99: 351-60.

13. Capelli M, Zuffetti F, Del Fabbro M, Testori T (2007) Immediate rehabilitation of the completely edentulous jaw with fixed prostheses supported by either upright or tilted implants: a multi center clinical study. Int J Oral Maxillofac Implants 22: 639-44.

14. Menini M, Signori A, Tealdo T, Bevilacqua M, Pera F, et al. (2012) Tilted implants in the immediate loading rehabilitation of the maxilla: A systematic review. J Dent Res 91: 821-7.

15. Graves S, Mahler BA, Javid B, Armellini D, Jensen OT (2011) Maxillary all-on-four therapy using angled implants: A 16-month clinical study of 1110 implants in 276 jaws. Oral Maxillofac Surg Clin North Am 23: 277-87.

16. Agliardi EL, Francetti L, Romeo D, Taschieri S, Del Fabbro M (2008) Immediate loading in the fully edentulous maxilla without bone grafting: the V-II-V technique. Minerva Stomatol 57: 251-63.

17. Calandriello R, Tomatis M (2005) Simplified treatment of the atrophic posterior maxilla via immediate/early function and tilted implants: A prospective 1-year clinical study. Clin Implant Dent Relat Res 7: S1-12.

18. Sorni M, Guarinós J, Garcia O, Peñarrocha M (2005) Implant rehabilitation of the atrophic upper jaw: A review of the literature since 1999. Med Oral Patol Oral Cir Bucal 10: 45-56.

19. Block MS, Haggerty CJ, Fisher GR (2009) Non grafting implant options for restoration of the edentulous maxilla. J Oral Maxillofac Surg 67: 872-81.

20. Del Fabbro M, Ceresoli V (2014) The fate of marginal bone around axial vs. tilted implants: a systematic review. Eur J Oral Implantol 2: S171-89. 
21. Cidade CP, Pimentel MJ, Amaral RC, Nóbilo MA, Barbosa JR (2014) Photoelastic analysis of all-on-four concept using different implants angulations for maxilla. Braz Oral Res 28: 1-7.

22. Krekmanov L, Kahn M, Rangert B, Lindström H (2002) Tilting of posterior mandibular and maxillary implants for improved prosthesis support. Int J Oral Maxillofac Implants 15: 405-14.

23. Maló P, Nobre Mde A, Petersson U, Wigren S (2006) A Pilot Study of Complete Edentulous Rehabilitation with Immediate Function Using a New Implant Design: Case Series. Clin Implant Dent Relat Res 8: 223-32.

24. Babbush CA, Hahn JA, Krauser JT, Rosenlicht JL (2011) The All on Four concept In: Dental Implants: The Art and Science (2 ${ }^{\text {nd }}$ Edn) St Louis, Mo: Saunders Elsevier, USA.

25. Van Lierde KM, Browaeys H, Corthals P, Matthys C, Mussche P, et al. (2012) Impact of fixed implant prosthetics using the 'all-on-four' treatment concept on speech intelligibility, articulation and oromyo functional behavior. Int J Oral Maxillo fac Surg 41: 1550-7.

26. Gherlone EF, Ferrini F, Crespi R, Gastaldi G, Capparé P (2015) Digital impressions for fabrication of definitive "all-on-four" restorations. Implant Dent 24: $125-9$.

27. Spinelli D, Ottria L, De Vico G, Bollero R, Barlattani A, et al. (2013) Full rehabilitation with nobel clinician $\left.{ }^{(}\right)$and procera implant bridge $\left({ }^{\oplus}\right)$ : case report. Oral Implantol 6: 25-36.

28. Ehsani S, Siadat H, Alikhasi M (2014) Comparative evaluation of impression accuracy of tilted and straight implants in All-on-Four technique. Implant Dent 23: $225-30$.

29. Dellavia C, Francetti L, Rosati R, Corbella S, Ferrario VF, et al. (2012) Electromyographic assessment of jaw muscles in patients with All-on-Four fixed implantsupported prostheses. J Oral Rehabil 39: 896-904.

30. Malo P, de Araujo Nobre M, Lopes A (2007) The use of computer guided flapless implant surgery and four implants placed in immediate function to support a fixed denture: preliminary results after a mean follow-up period of thirteen months. J Prosthet Dent 97: S26-34.

31. Zampelis A, Rangert B, Heijl L (2007) Tilting of splinted implants for improved prosthodontic support: A two-dimensional finite element analysis. J Prosthet Dent 97: 35-43.

32. Mupparapu M, Singer SR (2004) Implant imaging for the dentist. J Can Dent Assoc 70: 32.

33. Hassine MBH, Bucci P, Gasparro R, Lauro AED, Sammartino G (2015) Safe approach in “All-on-four” technique: A case report. Ann Stomatol (Roma) 5: 142-5.

34. Babbush CA (2012) Post-Treatment Quantification of Patient Experiences with Full-Arch Implant Treatment Using a Modification of the OHIP 14 Questionnaire. J Oral Implantol 38: 251-60.

35. Babbush CA, Kanawati A, Kotsakis GA (2016) Marginal Bone Stability Around Tapered, Platform-Shifted Implants Placed with an Immediately Loaded FourImplant-Supported Fixed Prosthetic Concept: A Cohort Study. Int J Oral Maxillofac Implants 31: 643-50.

36. Babbush CA, Kanawati A, Kotsakis GA, Hinrichs JE (2014) Patient-related and financial outcomes analysis of conventional full-arch rehabilitation versus the All-on-4 concept: A cohort study. Implant Dent 23: 218-24.

37. Bellini CM, Romeo D, Galbusera F, Taschieri S, Raimondi MT, et al. (2009) Comparison of tilted versus nontilted implant-supported prosthetic designs for the restoration of the edentulous mandible: A biomechanical study. Int J Oral Maxillofac Implants 24: 511-7.

38. Bellini CM, Romeo D, Galbusera F, Agliardi E, Pietrabissa R, et al. (2009) A finite element analysis of tilted versus nontilted implant configurations in the edentulous maxilla. Int J Prosthodont 22: 155-7.

39. Hussein MO, Rabie ME (2015) Three-dimensional nonlinear contact finite element analysis of mandibular all-on-four design. J Oral Implantol 41: e12-8.

40. Khatami AH, Smith CR (2008) “All-on-Four” Immediate Function Concept and Clinical Report of Treatment of an Edentulous Mandible with a Fixed Complete Denture and Milled Titanium Framework. J Prosthodont 17: 47-51.

41. Babbush CA, Kanawati A, Brokloff J (2013) A new approach to the All-on-Four treatment concept using narrow platform Nobel Active implants. J Oral Implantol 39: 314-25.

42. Wiltfang J, Schulte-Mosgau S, Nkenke E, Thorwarth M, Neukam FW, et al. (2005) Onlay augmentation versus sinus lift procedure in the treatment of the severely resorbed maxilla: A 5-year comparative longitudinal study. Int J Oral Maxillofac Surg 34: 885-9.

43. Dellavia C, Rosati R, Del Fabbro M, Pellegrini G (2014) Functional jaw muscle assessment in patients with a full fixed prosthesis on a limited number of implants: a review of the literature. Eur J Oral Implantol 7: 155-69.

44. Bevilacqua M, Tealdo T, Pera F, Menini M, Mossolov A, et al. (2008) Three-dimensional finite element analysis of load transmission using different implant inclinations and cantilever lengths. Int J Prosthodont 21: 539-42.

45. Takahashi T, Shimamura I, Sakurai K (2010) Influence of number and inclination angle of implants on stress distribution in mandibular cortical bone with All-on-four Concept. J Prosthod Res 54: 179-84.

46. Correa S, Ivancik J, Isaza JF, Naranjo M (2012) Evaluation of the structural behavior of three and four implant-supported fixed prosthetic restorations by finite element analysis. J Prosthodont Res 56: 110-9.

47. Patzelt SB, Bahat O, Reynolds MA, Strub JR (2014) The All-on-Four Treatment Concept: A Systematic Review. Clin Implant Dent Relat Res 16: 836-55.

48. Babbush CA, Kutsko GT, Brokloff J (2011) The all-on-four immediate function treatment concept with Nobel Active implants: a retrospective study. J Oral Implantol 37: 431-45.

49. Mozzati M, Arata V, Gallesio G, Musano F, Carossa S (2012) Immediate post extraction implant placement with immediate loading for maxillary full-arch rehabilitation. A two-year retrospective analysis. J Am Dent Assoc 143: 124-33.

50. Francetti L, Agliardi E, Testori T, Romeo D, Taschieri S, et al. (2008) Immediate rehabilitation of the mandible with fixed full prosthesis supported by axial and tilted implants: interim results of a single cohort prospective study. Clin Implant Dent Relat Res 10: 255-63.

51. Francetti L, Romeo D, Corbella S, Taschieri S, Del Fabbro M (2012) Bone level changes around axial and tilted implants in full-arch fixed immediate restorations. Interim results of a prospective study. Clin Implant Dent Relat Res 14: 646-54.

52. Francetti L, Corbella S, Taschieri S, Cavalli N, Del Fabbro M (2015) Medium- and Long-term complications in Full-Arch Rehabilitations Supported by Upright and Tilted Implants. Clin Implant Dent Relat Res 178: 758-64.

53. Nocini PF, Albanese M, Castellani R, Zanotti G, Canton L, et al. (2012) Application of the "All-on-Four"Concept and Guided Surgery in a Mandible Treated with a Free Vascularized Fibula Flap. J Cranio Fac Surg 23: e628-31. 


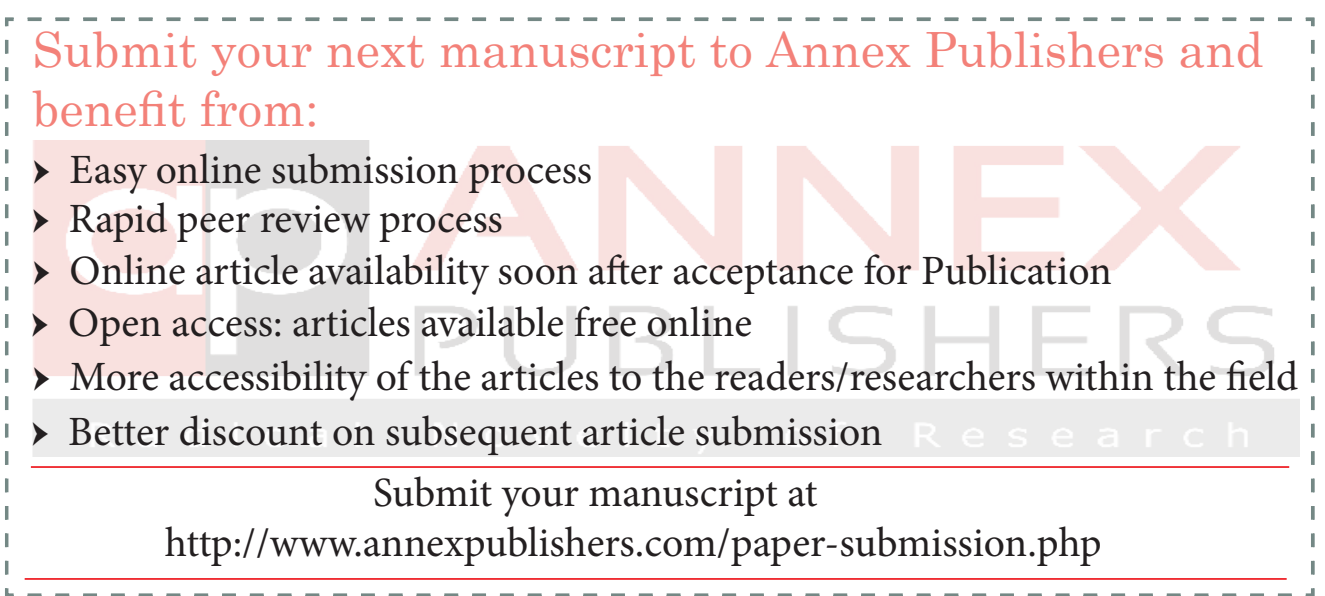

